\title{
Utility of Frailty Screening Tools in Older Surgical Patients
}

\author{
Alicia Wan Yan Poh, Shyh Poh Teo \\ Department of Internal Medicine, Raja Isteri Pengiran Anak Saleha (RIPAS) Hospital, Brunei Darussalam
}

Corresponding Author:

Shyh Poh Teo, FRACP

Department of Internal Medicine, Raja

Isteri Pengiran Anak Saleha (RIPAS)

Hospital, Bandar Seri Begawan BA

1710, Brunei Darussalam

E-mail: shyhpoh.teo@moh.gov.bn

ORCID:

https://orcid.org/0000-0002-6117-5774

Received: April 10, 2020

Revised: May 22, 2020

Accepted: May 28, 2020
Frailty is a loss of functional reserve that compromises a person's ability to cope with stressors such as surgery. Identifying and quantifying frailty may enable intensive rehabilitation interventions, caregiver support, or consideration of palliative care before surgery. This study describes the characteristics of five frailty screening tools, namely the Geriatric 8, Vulnerable Elders Survey-13, the Groningen Frailty Indicator, Edmonton Frailty Scale (EFS), and Clinical Frailty Scale. We further propose an approach incorporating a frailty scale into preoperative assessment, wherein older patients undergoing elective general surgery are screened using EFS, and frail patients are offered comprehensive geriatric assessment. The expected outcome is an individualized patient-centered care plan that will reduce frailty and optimize the patient's condition before surgery.

Key Words: Aged, Diagnosis, Frailty, General surgery, Risk assessment

\section{INTRODUCTION}

The international report "An Aging World: 2015" predicted that the number of people aged 65 years and above will increase from 617 million in 2015 to 1.6 billion by 2050, with two-thirds of the world's total older population living in Asia by 2050. ${ }^{1)}$ This older population will require planning for the provision of medical treatment, including surgery. Aging is a physiologic process in which there is deterioration in terms of cellular homeostasis, organ mass, and functional reserve. This loss of functional reserve varies among individuals and compromises their ability to cope with stressors such as surgery. ${ }^{2}$ Thus, it is important to quantify this variability or frailty to identify individuals at a significant risk of developing disability despite initial independence. ${ }^{3)}$

The vulnerability of a frail person is obvious when comparing their functional capacity to that of a non-frail person after physiological stress. For example, after minor surgery, an older person is expected to recover to their baseline function. However, a frail older person may experience a significant decline in function with increasing dependence or require rehabilitation to regain this func- tion. $^{4)}$

An observational study showed a poor prognosis for functional recovery among older patients hospitalized for acute medical illness and discharged with a new or additional disability. Identifying these patients early enables clinicians to provide intensive rehabilitation interventions, caregiver support, or consideration of palliative care. ${ }^{5)}$ For example, before surgical intervention, identifying frailty may facilitate early rehabilitation to improve the likelihood of recovery. It may also be appropriate to counsel frail patients against surgery or offer palliation if there is a high risk of complications, including dependence and mortality.

This paper provides an overview of frailty and frailty screening tools and suggests an approach that incorporates a frailty scale in preoperative assessments for older surgical patients to identify those who may benefit from early intervention and rehabilitation.

\section{FRAILTY}

The Asia-Pacific Clinical Practice Guideline indicates that frailty can be identified through three approaches, namely the Fried 
physical phenotype, the Rockwood and Mitnitski deficit accumulation model, and mixed physical and psychosocial models. ${ }^{6}$ Using the Fried phenotype, frailty is diagnosed when at least three of the following physical criteria are met: slow gait, low grip strength, weight loss, self-reported exhaustion, and low physical activity. While this approach is suitable for clinical settings, it requires time and equipment to assess grip strength and walking speed. ${ }^{6)}$ Another approach to quantify frailty is through calculating a frailty index based on cumulative health deficits from among at least 30 variables in multiple domains such as physical function, multi-morbidities, cognition, and psychosocial factors. ${ }^{6,7)}$ Both approaches for identifying frailty are predictive of disability, falls, functional decline, hospitalization, and risk with surgery, ${ }^{6-9)}$ implying flexibility in the choice of frailty model applied to older people. However, the frailty index may have a better ability to discriminate at the lower to middle end of the frailty continuum. ${ }^{9}$

Patients with chronic diseases at increased risks of surgical complications and decline in organ function. Quantifying frailty can a useful biomarker to predict surgical outcome and survival after surgery. ${ }^{10-13)}$ However, frailty indices do not differently weight specific comorbidities associated with worse surgical outcomes. For example, a retrospective study of 6,729 patients showed that chronic kidney disease, acute myocardial infarction, and intracerebral hemorrhage were the comorbidities most likely to be associated with post-anesthetic mortality. ${ }^{14)}$ The most common postoperative complications are delirium, pulmonary, and cardiac issues; which are mostly attributable to previous cardiovascular and cerebrovascular disease. ${ }^{15,16)}$ In addition, the relationship between age-related change in the immune system, inflammation and anemia leads to progressive loss of functional organ reserve and, eventually, frailty. ${ }^{17)}$

A patient's social circumstances are also important to consider, as social frailty significantly affects physical and cognitive frailty. Living alone, economic hardship, and limited social participation are associated with a decline in activities of daily living (ADL) and increased risk of disability. ${ }^{18)} \mathrm{A}$ hybrid approach including both physical and psychosocial models was recently adopted and incorporated into screening tools. ${ }^{6}$

The gold standard for frailty assessment is comprehensive geriatric assessment (CGA); it is usually performed by geriatricians and can take more than 1 hour to complete. Practically, it is more appropriate to screen using an assessment tool and request CGA for those who screen positive for frailty. Identifying frailty should result in a comprehensive care plan, addressing polypharmacy and contributors to weight loss, sarcopenia, and exhaustion in addition to a multi-component physical activity program and protein supplementation for malnourished patients. ${ }^{19)}$ Frailty screening tools are described briefly in the following section.

\section{FRAILTY SCREENING TOOLS}

Table 1 summarizes the main characteristics of five frailty screen-

Table 1. Frailty screening tools

\begin{tabular}{|c|c|c|c|c|c|}
\hline & \multicolumn{5}{|c|}{ Screening tests } \\
\hline & G8 & VES-13 & GFI & EFS & CFS \\
\hline Total number of items & 8 & 13 & 15 & 17 & $\mathrm{~N} / \mathrm{A}$ \\
\hline \multirow[t]{9}{*}{ Dimensions included in the tests } & Food intake & Reported health status & Mobility & Cognition & $\mathrm{N} / \mathrm{A}$ \\
\hline & Weight loss & Reported activities of dai- & Vision & General health status & \\
\hline & Mobility & ly living & Hearing & Functional independence & \\
\hline & Neuropsychological & Age & Nutrition & Social support & \\
\hline & problems & & Comorbidity & Medication use & \\
\hline & Body mass index & & Cognition & Nutrition & \\
\hline & Drugs & & Psychosocial & Mood & \\
\hline & Health status & & Reported physical fitness & Continence & \\
\hline & Age & & & Functional performance & \\
\hline Score range/categories & $0-17$ & $0-10$ & $0-15$ & $0-17$ & 1-9 categories \\
\hline \multirow[t]{3}{*}{ Cut-off value } & $\leq 14:$ frail & $\geq 3$ : increased risk of & $\geq 4:$ moderate or severe & $>7:$ increased risk of & Category 5: mild frailty \\
\hline & & death within 2 years & frailty & $\begin{array}{l}\text { postoperative complica- } \\
\text { tions }\end{array}$ & Category 9: terminally ill \\
\hline & & & & $\begin{array}{l}<4 \text { : lower risk of compli- } \\
\text { cations }\end{array}$ & \\
\hline Time to complete (min) & $\leq 5$ & 5 & 5 & $\leq 5$ & $<5$ \\
\hline
\end{tabular}

VES-13, Vulnerable Elders Survey-13; GFI, Groningen Frailty Indicator; EFS, Edmonton Frailty Scale; CFS, Clinical Frailty Scale. 
ing tools, namely the Geriatric 8 (G8), Vulnerable Elders Survey-13 (VES-13), Groningen Frailty Indicator (GFI), Edmonton Frailty Scale (EFS), and Clinical Frailty Scale (CFS). While these tools were mainly developed for community-dwelling older people, they may also be applicable in screening older surgical patients. The five frailty screening tools were identified through a literature search and shortlisted according to those that seemed practically applicable in the preoperative clinic assessment setting.

\section{G8}

The G8 was initially developed from the Mini-Nutritional Assessment-Short Form questionnaire specifically for patients with cancer. Two systematic reviews evaluating screening tools for frailty in older patients with cancer showed that compared with other screening tools, the G8 had the highest sensitivity but at the expense of specificity. ${ }^{20,21)}$ The high sensitivity of $\geq 80 \%$ was identified in six of eight studies using 14 points as a cut-off.

The G8 was modified to increase its specificity by adding 14 new items to the original questionnaire. The additional variables were asthenia, risk of fall, the Eastern Cooperative Oncology Group-Performance Status, urinary or fecal incontinence, heart failure or chronic heart disease, complete arrhythmia with atrial fibrillation, hypertension, diabetes mellitus, and chronic kidney disease. This improved the G8 specificity from $57.7 \%$ to $79 \%$ with a cutoff value of $\geq 6$ (or $88.4 \%$ with a cutoff value of $\geq 7$ ), while maintaining high specificity ( $87.2 \%$ in the original G8 compared with $89.2 \%$ in the modified G8 with a cutoff value of $\geq 6$ or $85.8 \%$ with a cutoff value of $\geq 7$ ). Compared with the original G8, the modified G8 was predictive of chemotherapy-related toxicity in cancers, including hematological cancers. ${ }^{22)}$

\section{VES-13}

The VES-13 was developed to screen community-dwelling older adults at risk of functional deterioration. It comprises 13 items, mainly regarding self-reported functional health. Generally, the VES-13 has better specificity than sensitivity, implying it may not be useful as a screening tool. Its sensitivity ranged from $39 \%-88 \%$, with $>80 \%$ in 2 of 11 studies, whereas its specificity ranged from $62 \%-100 \%$, with $100 \%$ in three of 11 studies. ${ }^{20,21)}$ The sensitivity and specificity can also be improved by using both the VES-13 and G8 screening tools concomitantly. ${ }^{21)}$

\section{GFI}

The GFI was designed for older adults in hospital, residential care, and community settings. Similar to the VES-13, the GFI has higher specificity ( $86 \%-87 \%)$ than sensitivity $(39 \%-66 \%)$. Both sensitivity (87\%) and specificity (70\%) were increased by lowering the standard cutoff value from $\geq 4$ to $\geq 3$. A cohort study found that frailty identified using the GFI was only associated with subjective postoperative reported limitations in terms of "daily activities" and "health problems"."2) Another study found that frail adults classified according to GFI required more assistance in ADLs, were more complex patients with higher comorbidities, and experienced lower satisfaction and quality of life. ${ }^{24)}$ However, these results of these studies were consistent with those of a systematic review reporting that neither VES-13 nor GFI was associated with postoperative morbidity and mortality. ${ }^{21)}$

\section{EFS}

The EFS is another frailty screening tool for older people that can be completed rapidly. Unlike other screening tools, it incorporates functional performance using the Timed Up and Go (TUG) test, social support, and cognition screening with the clock-drawing test. These components may be used to identify important issues that need to be addressed through CGA. Higher TUG predicted the risk of early mortality in oncology patients receiving chemotherapy, postoperative complications, and morbidity. ${ }^{21,25,26)}$ TUG also assesses balance, gait, and (indirectly) risk of falls, which may identify patients who would benefit from preoperative rehabilitation. $^{27)}$

Surgical complications may result in negative psychosocial effects that are associated with prolonged wound healing and impaired immune function. Limited postoperative social support for older people may affect their recovery. ${ }^{28,29)}$ Cognitive screening using the clock-drawing test predicted patients who were likely to develop postoperative delirium and mortality. ${ }^{30)}$ A study screening for frailty in community-dwelling older adults showed that the clock-drawing test component of the EFS had high sensitivity (82.6\%, specificity $36.9 \%$ ) in identifying cognitive impairment. ${ }^{31}$ Overall, EFS score $>7$ was associated with postoperative complications and increased hospital stay. ${ }^{15,22)}$

\section{CFS}

The CFS is used to provide a common language among health professionals to define frailty. This tool describes frailty with scores ranging from 1 (very fit) to 7 (completely dependent). It was initially developed from a prospective cohort study, the Canadian Study of Health and Aging, and validated in patients followed up over 5 years. Additional categories were added subsequently for a 
total of seven to differentiate terminally ill but independent patients from those dependent on others for ADLs. ${ }^{32)}$

Overall, CFS is an easy and quick test that does not require physical performance tests such as TUG or hand grip. It is also predictive of the duration of hospital stay and risk of death. ${ }^{33)}$ However, the CFS requires subjective clinical judgment, which may lead to problems with inter-rater reliability. The frailty scores are also heavily weighted on a person's ability to perform basic and instrumental ADLs and do not cover other aspects such as cognition and psychosocial assessment that may impact preoperative management.

\section{FRAILTY IN OLDER SURGICAL PATIENTS}

Studies on older surgical patients have also consistently shown the association between frailty and poor surgical outcomes. Thus, frailty screening may be useful as a risk-stratification tool specifically in older surgical patients. For example, frailty screening using a modified frailty index for elective total shoulder arthroplasty showed that frailty was more predictive of postoperative complications, readmission, reoperation, and increased length of stay than age alone. $^{34)}$

A cohort study of non-cardiac surgical patients used a novel operative stress score to quantify physiologic stress for surgical procedures. Using the Risk Analysis Index for frailty screening, the study found that frail patients had higher rates of complications and 30day mortality, which continually increased at 90 and 180 days regardless of physiological stress scores, where even low- to moderate-stress procedures such as cystoscopy were high-risk in frail patients. $^{35)}$

A study comparing the diagnostic accuracy of six screening instruments for frailty, including the VES-13, G8, and GFI, in older patients undergoing emergency abdominal surgery found that these screening tools were predictive of postoperative outcomes, with the VES-13 showing the highest sensitivity and negative predictive value for postoperative morbidity and mortality. ${ }^{36)}$ Another study on emergency abdominal surgery combined the Surgical Apgar Score, which predicts postoperative mortality based on estimated blood loss, blood pressure, and heart rate, with the G8 for frailty screening. This study reported that both measures were independent predictors of postoperative adverse events and proposed the use of both scores to predict outcomes. ${ }^{37)}$

The application of the GFI to older patients undergoing vascular surgery showed higher complication rates, 30-day mortality, and discharge to residential care in frail patients. ${ }^{38)}$ Frailty identified using the modified frailty index and GFI was also consistently associated with poor outcomes in terms of length of stay, mortality, com- plications, and discharge to residential care for head and neck surgery. $^{39)}$

Retrospective analyses of prospective databases of elective spine surgery also confirmed positive correlations between frailty and mortality, postoperative complications, and length of stay. While the modified frailty index was recommended, the researchers suggested the use of more comprehensive frailty indices specific for spinal surgery, such as the Adult Spinal Deformity frailty index, and consideration of adding clinical, radiographic, and laboratory measures to improve outcome predictions. ${ }^{40)}$ Another study that recalibrated and validated the Risk Analysis Index using a national surgical registry reported that the revision improved its discriminatory ability to predict mortality. ${ }^{41)}$

Based on currently available studies on older surgical patients, frailty screening appears to discriminate and identify high-risk patients regardless of screening tool and type of surgery. The ability to predict outcomes may be improved by combining frailty screening tools with other tools (such as the Surgical Apgar Score), adding more variables to achieve a more comprehensive frailty index, or adapting and validating available frailty tools to individual surgical interventions or clinical settings.

However, in terms of feasibility and practical implementation of frailty screening for older surgical patients, it is more appropriate to integrate a common frailty screening tool for surgical patients that can be applied during pre-assessment in clinics.

\section{JOURNEY FROM REFERRAL TO SURGERY}

While the referral pathway to surgery may vary depending on clinical settings, it generally begins when patients are provided the option of surgery at the time of diagnosis in the clinic. For most elective surgical interventions, patients attend preoperative assessments by anesthetists $1-2$ weeks before surgery. When issues are identified, the patients may be referred to organ-specific specialists for consultation. This limited engagement between patients and doctors and the possible need for interventions to optimize the patient's condition before surgery tends to be done within a limited time, with occasional consequent postponement of surgery. This is consistent with the literature, suggesting it can take up to 50 days from a general practitioner referral to the first formal assessment, which may be 2-14 days before the day of surgery. ${ }^{42}$

The preoperative assessment pathway has two main roles: first, to ensure discussion and appropriate decision-making regarding surgery, and second, to ensure the patient is as prepared as possible to maximize their resilience to the psychological stresses of surgery. From the anesthetist's perspective, this pathway can be re-engineered so that risk stratification occurs through simple screening 
tools, then staged early using cardiopulmonary tests as an objective physiological assessment, with interventions if necessary before surgery. ${ }^{42)}$ Similarly, for older patients undergoing elective surgery, screening tools may be utilized to identify frailty and such patients can be referred preoperatively for interventions to optimize their recovery.

\section{SUGGESTED APPROACH: PREOPERATIVE ASSESSMENT INCORPORATING A FRAILTY SCALE}

A suggested approach for preoperative assessment incorporating a frailty scale is summarized in Fig. 1. In this approach, all patients aged 65 years and older undergoing elective general abdominal surgery are screened using the EFS during the surgical consulta- tion, as soon as a decision for surgery is made. This should be done at least 3 months before surgery to allow sufficient time for interventions. The EFS is multi-domain; hence, it can be used to identify areas that require further assessment. Moreover, the assessment can be performed quickly in clinics by non-geriatricians.

Although most of the frailty tools appear to be predictive of poor surgical outcomes, with some differences in sensitivity and specificity, the EFS was preferred over the other tools as it incorporates objective cognitive and functional performance through the TUG and clock-drawing tests within the short time required to complete the tool. These objective measures may avoid potential variability in self-reporting among older surgical patients.

Patients with EFS scores $>7$ should be referred for CGA. As there is usually limited time before the date of surgery, coordina-

Older adults aged 65 and above diagnosed with abdominal cancer

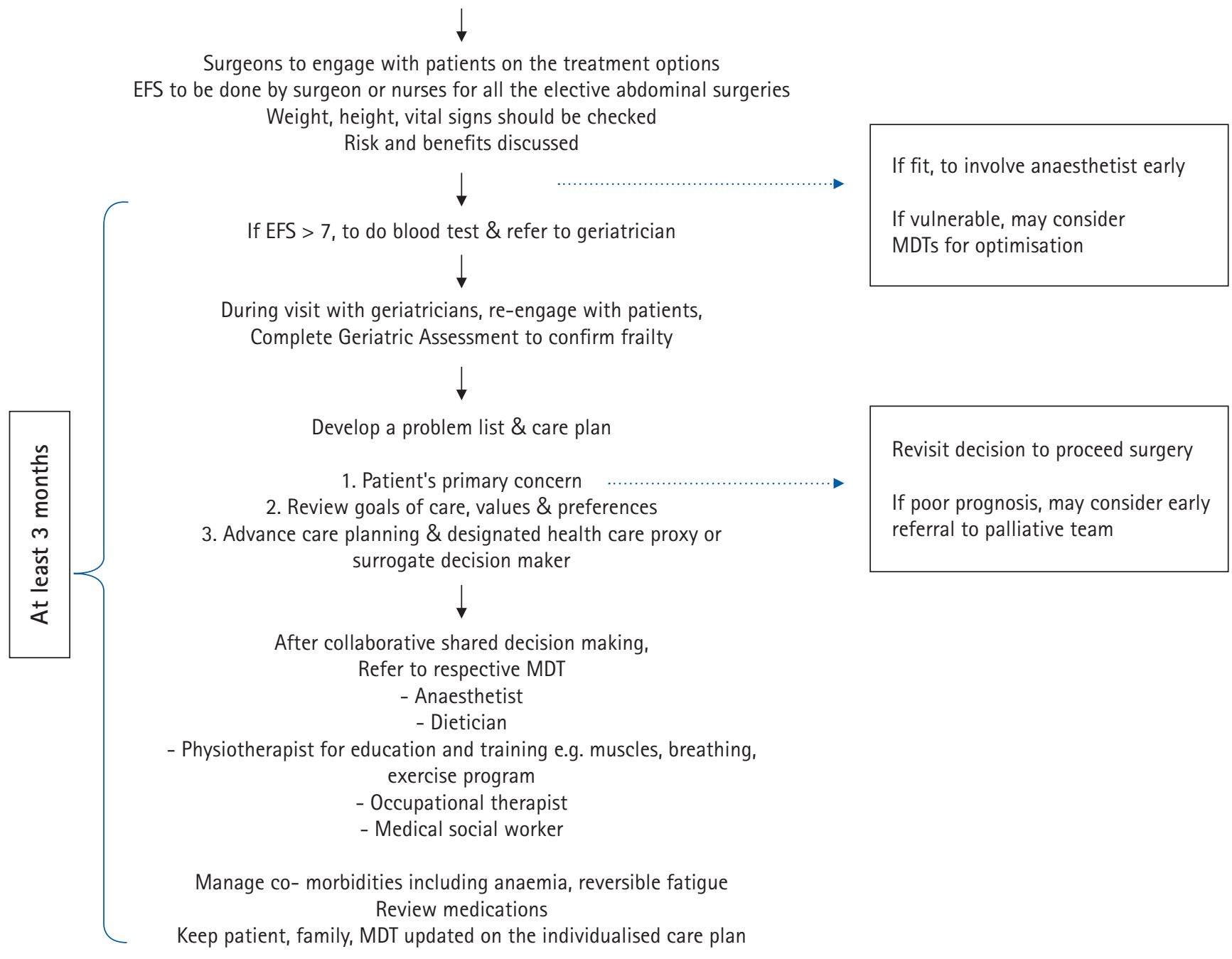

Fig. 1. A suggested approach for preoperative assessment incorporating a frailty scale. EFS, Edmonton frailty scale; MDT, multidisciplinary team 
tion of assessment and treatment by a multidisciplinary team is required. This team should comprise surgeons, geriatricians, organ-specific physicians, anesthetists, physiotherapists, occupational therapists, dietitians, social workers, nurses, and patients. For patients with a poor prognosis, early referral to palliative teams for supportive care is warranted. The main outcome is individualized patient-centered care plans that are actionable and feasible and which consider the patient's personal treatment goals and preferences. Nutritional, physical, cognitive, and combination interventions can significantly reduce frailty in older people, which is beneficial preoperatively. ${ }^{43)}$

The possible postoperative recovery trajectories should be discussed and understood by patients and families to allow proactive planning for potential care needs on discharge. Advance care planning should also be discussed, and a designated decisionmaker or health care proxy may need to be documented in patients' medical records. $^{44)}$

\section{CONCLUSION}

Frailty is associated with complications, prolonged recovery after surgery, and mortality. Frailty screening tools are available to identify frail patients that may benefit from CGA and individualized management plans to optimize patient conditions before surgery. We have proposed an algorithm for frailty screening that appears to be feasible to implement in preoperative assessment clinics. Further research is required to assess whether this approach will improve outcomes in older people.

\section{ACKNOWLEDGMENTS}

\section{CONFLICT OF INTEREST}

The researchers claim no conflicts of interest.

\section{AUTHOR CONTRIBUTIONS}

Conceptualization, AWP, SPT; Data curation, AWP, SPT; Investigation, AWP, SPT; Writing-original draft, AWP, SPT; Writing-review \& editing, AWP, SPT.

\section{REFERENCES}

1. He W, Goodkind D, Kowal P. An aging world: 2015 (International Population Reports). Washington, DC: U.S. Government Publishing Office; 2016.

2. Nigam Y, Knight J, Bhattacharya S, Bayer A. Physiological changes associated with aging and immobility. J Aging Res 2012;2012:468469.
3. Biganzoli L, Mislang AR, Di Donato S, Becheri D, Biagioni C, Vitale S, et al. Screening for frailty in older patients with early-stage solid tumors: a prospective longitudinal evaluation of three different geriatric tools. J Gerontol A Biol Sci Med Sci 2017;72:922-8.

4. Clegg A, Young J, Iliffe S, Rikkert MO, Rockwood K. Frailty in elderly people. Lancet 2013;381:752-62.

5. Boyd CM, Landefeld CS, Counsell SR, Palmer RM, Fortinsky $\mathrm{RH}$, Kresevic D, et al. Recovery of activities of daily living in older adults after hospitalization for acute medical illness. J Am Geriatr Soc 2008;56:2171-9.

6. Dent E, Lien C, Lim WS, Wong WC, Wong CH, Ng TP, et al. The Asia-Pacific clinical practice guidelines for the management of frailty. J Am Med Dir Assoc 2017; 18:564-75.

7. Rockwood K, Blodgett JM, Theou O, Sun MH, Feridooni HA, Mitnitski A, et al. A frailty index based on deficit accumulation quantifies mortality risk in humans and in mice. Sci Rep 2017;7:43068.

8. Li G, Thabane L, Ioannidis G, Kennedy C, Papaioannou A, Adachi JD. Comparison between frailty index of deficit accumulation and phenotypic model to predict risk of falls: data from the global longitudinal study of osteoporosis in women (GLOW) Hamilton cohort. PLoS One 2015;10:e0120144.

9. Blodgett J, Theou O, Kirkland S, Andreou P, Rockwood K. Frailty in NHANES: Comparing the frailty index and phenotype. Arch Gerontol Geriatr 2015;60:464-70.

10. Kristjansson SR, Nesbakken A, Jordhoy MS, Skovlund E, Audisio RA, Johannessen $\mathrm{HO}$, et al. Comprehensive geriatric assessment can predict complications in elderly patients after elective surgery for colorectal cancer: a prospective observational cohort study. Crit Rev Oncol Hematol 2010;76:208-17.

11. Ommundsen N, Wyller TB, Nesbakken A, Jordhoy MS, Bakka A, Skovlund E, et al. Frailty is an independent predictor of survival in older patients with colorectal cancer. Oncologist 2014;19:1268-75.

12. Robinson TN, Wu DS, Pointer L, Dunn CL, Cleveland JC Jr, Moss M. Simple frailty score predicts postoperative complications across surgical specialties. Am J Surg 2013;206:544-50.

13. Makary MA, Segev DL, Pronovost PJ, Syin D, Bandeen-Roche K, Patel P, et al. Frailty as a predictor of surgical outcomes in older patients. J Am Coll Surg 2010;210:901-8.

14. Chu CL, Chiou HY, Chou WH, Chang PY, Huang YY, Yeh HM. Leading comorbidity associated with 30-day post-anesthetic mortality in geriatric surgical patients in Taiwan: a retrospective study from the health insurance data. BMC Geriatr 2017; $17: 245$

15. Dasgupta M, Rolfson DB, Stolee P, Borrie MJ, Speechley M. 
Frailty is associated with postoperative complications in older adults with medical problems. Arch Gerontol Geriatr 2009; 48:78-83.

16. Sieber FE, Barnett SR. Preventing postoperative complications in the elderly. Anesthesiol Clin 2011;29:83-97.

17. Rohrig G. Anemia in the frail, elderly patient. Clin Interv Aging 2016;11:319-26.

18. Makizako H, Tsutsumimoto K, Shimada H, Arai H. Social frailty among community-dwelling older adults: recommended assessments and implications. Ann Geriatr Med Res 2018;22:3-8.

19. Dent E, Morley JE, Cruz-Jentoft AJ, Woodhouse L, Rodriguez-Manas L, Fried LP, et al. Physical frailty: ICFSR International clinical practice guidelines for identification and management. J Nutr Health Aging 2019;23:771-87.

20. Hamaker ME, Jonker JM, de Rooij SE, Vos AG, Smorenburg $\mathrm{CH}$, van Munster BC. Frailty screening methods for predicting outcome of a comprehensive geriatric assessment in elderly patients with cancer: a systematic review. Lancet Oncol 2012;13: e437. -44.

21. Decoster L, Van Puyvelde K, Mohile S, Wedding U, Basso U, Colloca G, et al. Screening tools for multidimensional health problems warranting a geriatric assessment in older cancer patients: an update on SIOG recommendations. Ann Oncol 2015;26:288-300.

22. Martinez-Tapia C, Canoui-Poitrine F, Bastuji-Garin S, Soubeyran P, Mathoulin-Pelissier S, Tournigand C, et al. Optimizing the G8 screening tool for older patients with cancer: diagnostic performance and validation of a six-item version. Oncologist 2016;21: 188-95.

23. Bras L, Peters TT, Wedman J, Plaat BE, Witjes MJ, van Leeuwen BL, et al. Predictive value of the Groningen Frailty Indicator for treatment outcomes in elderly patients after head and neck, or skin cancer surgery in a retrospective cohort. Clin Otolaryngol 2015;40:474-82.

24. Peters LL, Boter H, Buskens E, Slaets JP. Measurement properties of the Groningen Frailty Indicator in home-dwelling and institutionalized elderly people. J Am Med Dir Assoc 2012; 13:546-51.

25. Soubeyran P, Fonck M, Blanc-Bisson C, Blanc JF, Ceccaldi J, Mertens C, et al. Predictors of early death risk in older patients treated with first-line chemotherapy for cancer. J Clin Oncol 2012;30:1829-34.

26. Huisman MG, van Leeuwen BL, Ugolini G, Montroni I, Spiliotis J, Stabilini C, et al. "Timed Up \& Go": a screening tool for predicting 30-day morbidity in onco-geriatric surgical patients? A multicenter cohort study. PLoS One 2014;9:e86863.

27. Kronzer VL, Jerry MR, Ben Abdallah A, Wildes TS, Stark SL,
McKinnon SL, et al. Preoperative falls predict postoperative falls, functional decline, and surgical complications. EBioMedicine 2016;12:302-8.

28. Pinto A, Faiz O, Davis R, Almoudaris A, Vincent C. Surgical complications and their impact on patients' psychosocial well-being: a systematic review and meta-analysis. BMJ Open 2016;6:e007224.

29. Kiecolt-Glaser JK, Page GG, Marucha PT, MacCallum RC, Glaser R. Psychological influences on surgical recovery: perspectives from psychoneuroimmunology. Am Psychol 1998;53:1209-18.

30. Long LS, Shapiro WA, Leung JM. A brief review of practical preoperative cognitive screening tools. Can J Anaesth 2012;59:798804.

31. Fabricio-Wehbe SC, Schiaveto FV, Vendrusculo TR, Haas VJ, Dantas RA, Rodrigues RA. Cross-cultural adaptation and validity of the 'Edmonton Frail Scale - EFS' in a Brazilian elderly sample. Rev Lat Am Enfermagem 2009; 17:1043-9.

32. Rockwood K, Song X, MacKnight C, Bergman H, Hogan DB, McDowell I, et al. A global clinical measure of fitness and frailty in elderly people. CMAJ 2005;173:489-95.

33. Juma S, Taabazuing MM, Montero-Odasso M. Clinical frailty scale in an acute medicine unit: a simple tool that predicts length of stay. Can Geriatr J 2016;19:34-9.

34. Holzgrefe RE, Wilson JM, Staley CA, Anderson TL, Wagner ER, Gottschalk MB. Modified frailty index is an effective risk-stratification tool for patients undergoing total shoulder arthroplasty. J Shoulder Elbow Surg 2019;28:1232-40.

35. Shinall MC Jr, Arya S, Youk A, Varley P, Shah R, Massarweh NN, et al. Association of preoperative patient frailty and operative stress with postoperative mortality. JAMA Surg 2019; 155:e194620.

36. Kenig J, Zychiewicz B, Olszewska U, Barczynski M, Nowak W. Six screening instruments for frailty in older patients qualified for emergency abdominal surgery. Arch Gerontol Geriatr 2015;61:437-42.

37. Kenig J, Mastalerz K, Lukasiewicz K, Mitus-Kenig M, Skorus U. The Surgical Apgar Score predicts outcomes of emergency abdominal surgeries both in fit and frail older patients. Arch Gerontol Geriatr 2018;76:54-9.

38. Visser L, Banning LBD, El Moumni M, Zeebregts CJ, Pol RA. The effect of frailty on outcome after vascular surgery. Eur J Vasc Endovasc Surg 2019;58:762-9.

39.Fu TS, Sklar M, Cohen M, de Almeida JR, Sawka AM, Alibhai $\mathrm{SMH}$, et al. Is frailty associated with worse outcomes after head and neck surgery? A narrative review. Laryngoscope 2020; 130:1436-42.

40. Simcox T, Antoku D, Jain N, Acosta F, Hah R. Frailty syndrome 
and the use of frailty indices as a preoperative risk stratification tool in spine surgery: a review. Asian Spine J 2019;13:861-73.

41. Arya S, Varley P, Youk A, Borrebach JA, Perez S, Massarweh NN, et al. Recalibration and external validation of the risk analysis index: a surgical frailty assessment tool. Ann Surg 2019;Mar 19; [Epub]. http://doi.org/10.1097/SLA. 0000000000003276.

42. Grocott MPW, Plumb JOM, Edwards M, Fecher-Jones I, Levett DZH. Re-designing the pathway to surgery: better care and added value. Perioper Med (Lond) 2017;6:9.
43. Ng TP, Feng L, Nyunt MS, Feng L, Niti M, Tan BY, et al. Nutritional, physical, cognitive, and combination interventions and frailty reversal among older adults: a randomized controlled trial. Am J Med 2015;128:1225-36.

44. Mohanty S, Rosenthal RA, Russell MM, Neuman MD, Ko CY, Esnaola NF. Optimal perioperative management of the geriatric patient: a best practices guideline from the American College of Surgeons NSQIP and the American Geriatrics Society. J Am Coll Surg 2016;222:930-47. 